\title{
Hermite function description of Feichtinger's space S0
}

\author{
Citation for published version (APA): \\ Janssen, A. J. E. M. (2005). Hermite function description of Feichtinger's space S0. Journal of Fourier Analysis \\ and Applications, 11(5), 577-588. https://doi.org/10.1007/s00041-005-4077-y
}

DOI:

10.1007/s00041-005-4077-y

Document status and date:

Published: 01/01/2005

\section{Document Version:}

Publisher's PDF, also known as Version of Record (includes final page, issue and volume numbers)

\section{Please check the document version of this publication:}

- A submitted manuscript is the version of the article upon submission and before peer-review. There can be important differences between the submitted version and the official published version of record. People interested in the research are advised to contact the author for the final version of the publication, or visit the DOI to the publisher's website.

- The final author version and the galley proof are versions of the publication after peer review.

- The final published version features the final layout of the paper including the volume, issue and page numbers.

Link to publication

\section{General rights}

Copyright and moral rights for the publications made accessible in the public portal are retained by the authors and/or other copyright owners and it is a condition of accessing publications that users recognise and abide by the legal requirements associated with these rights.

- Users may download and print one copy of any publication from the public portal for the purpose of private study or research.

- You may not further distribute the material or use it for any profit-making activity or commercial gain

- You may freely distribute the URL identifying the publication in the public portal.

If the publication is distributed under the terms of Article $25 \mathrm{fa}$ of the Dutch Copyright Act, indicated by the "Taverne" license above, please follow below link for the End User Agreement:

www.tue.nl/taverne

\section{Take down policy}

If you believe that this document breaches copyright please contact us at:

openaccess@tue.nl

providing details and we will investigate your claim. 
The Journal of Fourier Analysis and Applications

Volume 11, Issue 5, 2005

\title{
Hermite Function Deseription of Feichtinger's Space $S_{0}$
}

\author{
A. J. E. M. Janssen
}

Communicated by Hans G. Feichtinger

\begin{abstract}
For $\gamma \in \mathbb{R}$ we let $C(\gamma)$ be the set of all $f \in \mathcal{S}^{\prime}$ for which $\sum_{n=0}^{\infty}\left|\left(f, h_{n}\right)\right|(n+1)^{\gamma}<$ $\infty$, where $\left(h_{n}\right)_{n=0,1, \ldots}$ is the orthonormal base of Hermite functions. We show that $C(\gamma) \subset S_{0}$ if and only if $\gamma \geq \frac{1}{4}$ and that $S_{0} \subset C(\gamma)$ if and only if $\gamma \leq-\frac{1}{4}$. Here $S_{0}$ is Feichtinger's space.
\end{abstract}

\section{Introduction}

Feichtinger's space $S_{0}$ is the largest Banach space of functions of a real variable that allows a relatively carefree development of time-frequency analysis and, in particular, Gabor analysis, see [1], Chapter 3. There are many equivalent characterizations of the space $S_{0}$. For our purposes, it is most convenient to work with the description in terms of the Gaussian window Short-Time Fourier Transform (STFT) of the members of $S_{0}$. Hence, letting for $x, y \in \mathbb{R}$

$$
g_{x, y}(t)=2^{1 / 4} \exp \left(-\pi(t-x)^{2}+2 \pi i y t\right), \quad t \in \mathbb{R},
$$

we say that an $f \in L^{2}$ belongs to $S_{0}$ when

$$
\|f\|_{S_{0}}:=\int_{-\infty}^{\infty} \int_{-\infty}^{\infty}\left|\left(f, g_{x, y}\right)\right| d x d y<\infty
$$

In phase space analysis and in the time-frequency analysis of functions in $L^{2}(\mathbb{R})$, the orthonormal base $\left(h_{n}\right)_{n=0,1, \ldots}$ for $L^{2}(\mathbb{R})$ of Hermite functions plays an important role, see [2], Chapter 1, Section 7, [3], Section 3.4. In this article, we aim at describing the space

Math Subject Classifications. Primary 42C15; secondary 42B35, 33C45, 42A55.

Keywords and Phrases. Feichtinger's space, Hermite functions, time-frequency analysis, sequence space, Bargmann transform. 
$S_{0}$ in terms of the Hermite coefficients $\left(f, h_{n}\right)$ of its members $f$. To be more precise, we let for $\gamma \in \mathbb{R}$

$$
C(\gamma):=\left\{f \in \mathcal{S}^{\prime}\left|\sum_{n=0}^{\infty}\right|\left(f, h_{n}\right) \mid(n+1)^{\gamma}<\infty\right\},
$$

where $\mathcal{S}^{\prime}$ is the set of tempered distributions (dual of Schwartz's $\mathcal{S}$ ). We shall show that $C\left(\frac{1}{4}\right) \subset S_{0} \subset C\left(-\frac{1}{4}\right)$ and that $C(\gamma) \not \subset S_{0}$ when $\gamma<\frac{1}{4}$ and $S_{0} \not \subset C(\gamma)$ when $\gamma>-\frac{1}{4}$. Figure 1 summarizes the situation. It may be noted here that from [4] one has $\mathcal{S}=$ $\bigcap_{\gamma \in \mathbb{R}} C(\gamma)$ while $\mathcal{S}^{\prime}=\bigcup_{\gamma \in \mathbb{R}} C(\gamma)$. The main tools we use to prove these results are the Bargmann transform that allows explicit computations with the Gaussian window STFTs of the Hermite functions, and Mehler's formula. The inclusions $C\left(\frac{1}{4}\right) \subset S_{0} \subset$ $C\left(-\frac{1}{4}\right)$ are rather straightforward consequences of the explicit formulas for $\left(h_{n}, g_{x, y}\right)$ and orthonormality of the $h_{n}, n=0,1, \ldots$ For the proof of $S_{0} \not \subset C(\gamma)$, certain manipulations with Mehler's formula are required, while the proof of $C(\gamma) \not \subset S_{0}$ relies on a consideration of certain "lacunary" Hermite series $\sum_{m} c_{m} h_{A^{m}}$ for which one has $\left\|\sum_{m} c_{m} h_{A^{m}}\right\|_{S_{0}} \approx$ $\sum_{m}\left|c_{m}\right|\left\|h_{A^{m}}\right\|_{S_{0}}$.

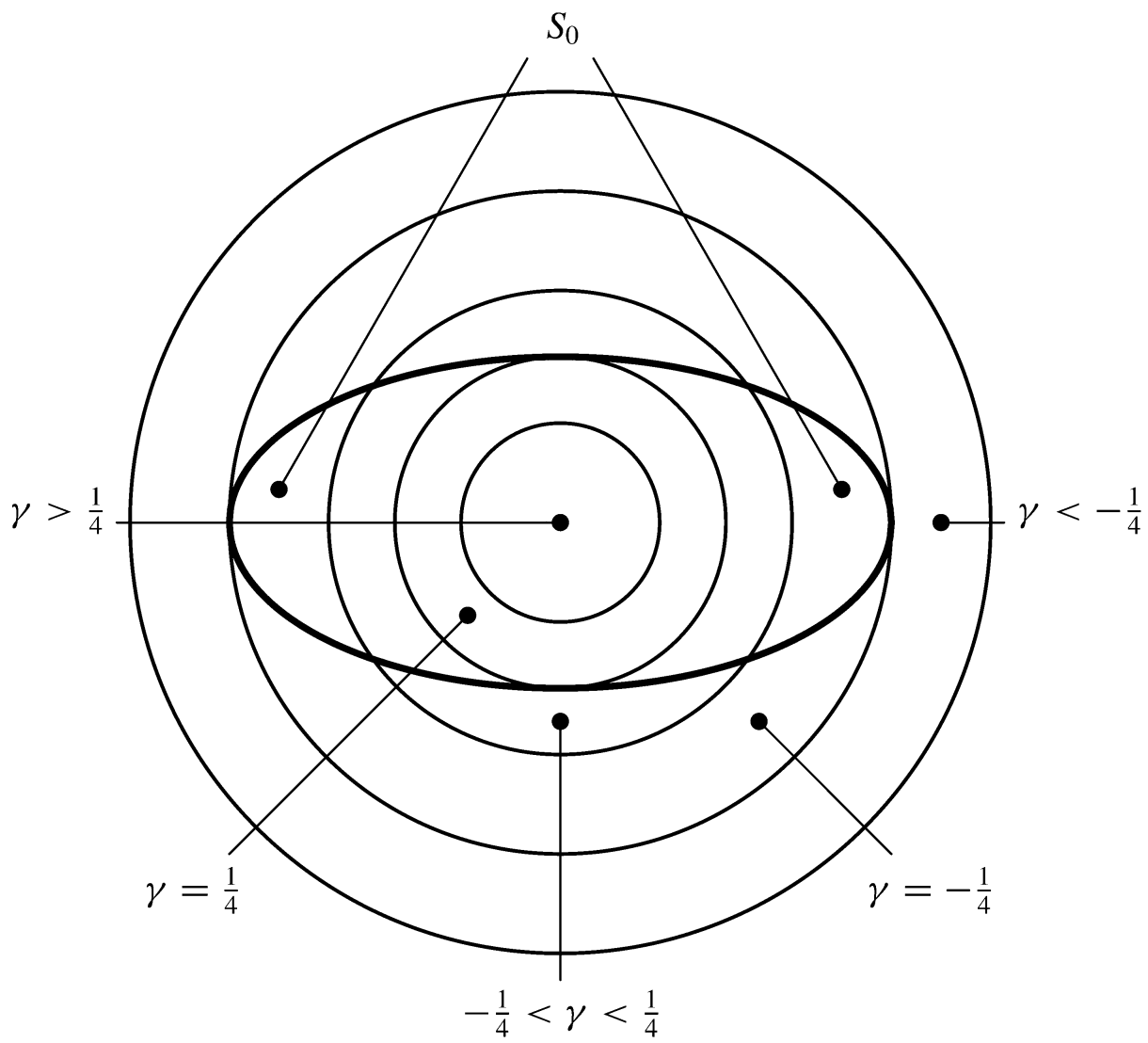

FIGURE 1 Positioning of $S_{0}$ relative to $C(\gamma), \gamma \in \mathbb{R}$. 
Formula (2.9) for the Gaussian window STFT of the Hermite functions is crucial in these investigations. This formula shows, for instance, that the $n^{\text {th }}$-Hermite function can be thought as being located in the time-frequency plane near the circle around 0 of radius $((n+1) / \pi)^{1 / 2}$. Furthermore, as a function of $\varphi=\arg (x-i y)$ for a fixed value of $r=\left(x^{2}+y^{2}\right)^{1 / 2}$, the collection of functions $\left(h_{n}, g_{x, y}\right), n=0,1, \ldots$, is orthogonal. These circumstances lead one to expect quite transparent characterization results for modulation spaces $M_{m}^{p, q}$, see [3], Definition 11.1.2 on p. 219 and 11.3.1 on p. 231, with $p=q=2$ and radially symmetric, well-behaved weight functions $m(x, y)=K\left(\pi\left(x^{2}+y^{2}\right)\right)$. Indeed, when, say $f \in \mathcal{S}$ and $K$ has at most polynomial growth, one has the formula

$$
\begin{gathered}
\int_{-\infty}^{\infty} \int_{-\infty}^{\infty}\left|\left(f, g_{x, y}\right)\right|^{2} K\left(\pi\left(x^{2}+y^{2}\right)\right) d x d y \\
=\sum_{n=0}^{\infty}\left|\left(f, h_{n}\right)\right|^{2} \int_{0}^{\infty} \frac{e^{-v} v^{n}}{n !} K(v) d v
\end{gathered}
$$

for the square of the $M_{m}^{2,2}$-norm of $f$. This follows readily from the above observations on the $\left(h_{n}, g_{x, y}\right)$ and the completeness and orthonormality of the $h_{n}$ in $L^{2}(\mathbb{R})$; also see [5], Theorem 5.5.5 on p. 220 for a detailed proof of (1.4). When, in addition, $K$ is slowly varying (a notion that can be made more precise in a similar way as is done in [6], Definition 3 on p. 284), one can effectively replace the integrals on the second line of (1.4) by $K(n+1)$. Thus, we arrive at the characterization result

$$
f \in M_{m}^{2,2} \Leftrightarrow \sum_{n=0}^{\infty}\left|\left(f, h_{n}\right)\right|^{2} K(n+1)<\infty
$$

a result that is similar in spirit to those that can be found in [6], Section 4.

The results for $S_{0}$ presented above show that a characterization of $S_{0}$ in terms of a single requirement on the Hermite coefficients of its members, such as is the case for $M_{m}^{2,2}$ spaces with radially symmetric weights $m$ of certain type via (1.5), cannot be expected to exist. This is no surprise since orthonormality is a notion that one normally associates with $L^{2}$-norms while the norm on $S_{0}$ is an $L^{1}$-norm, see (1.2). One has that $S_{0}$ coincides with the modulation space $M_{m \equiv 1}^{p=1, q=1}$. The results of this article on $S_{0}$ thus show to what extent the crisp result in (1.5) gets detuned when one passes from 2-norms to 1-norms at either side of the $\Leftrightarrow$ in (1.5) ( $K \equiv 1$ here). The proofs of the results get more complicated as well, but the concentration result for the Gaussian STFT of the $h_{n}$ 's is still crucial.

In Section 2 we develop notations, normalizations and relevant formulas, such as the one for $\left(h_{n}, g_{x, y}\right)$ and Mehler's formula, concerning the Hermite functions. In Section 3 we show the inclusions $C\left(\frac{1}{4}\right) \subset S_{0} \subset C\left(-\frac{1}{4}\right)$. In Section 4 we show that $S_{0} \not \subset C(\gamma)$ when $\gamma>-\frac{1}{4}$ and in Section 5 we show that $C(\gamma) \not \subset S_{0}$ when $\gamma<\frac{1}{4}$.

\section{Preliminaries About Hermite Functions}

We follow the notations and normalizations of [2], p. 51; these are identical with those in [3], Section 3.4 and [7], except that in [3] and [7] one writes $H_{n}$ and $\psi_{n}$, respectively, 
for $h_{n}$. Thus, we let

$$
h_{n}(t)=\frac{2^{1 / 4}}{\sqrt{n !}}\left(\frac{-1}{2 \sqrt{\pi}}\right)^{n} e^{\pi t^{2}}\left(\frac{d}{d t}\right)^{n} e^{-2 \pi t^{2}}, \quad n=0,1, \ldots, t \in \mathbb{C},
$$

be the Hermite functions. In terms of the Hermite polynomials

$$
H_{n}(x)=(-1)^{n} e^{x^{2}}\left(\frac{d}{d x}\right)^{n} e^{-x^{2}}, \quad n=0,1, \ldots, x \in \mathbb{C},
$$

one has

$$
h_{n}(t)=\left(2^{n-1 / 2} n !\right)^{-1 / 2} H_{n}(t \sqrt{2 \pi}) e^{-\pi t^{2}}, \quad n=0,1, \ldots, t \in \mathbb{C} .
$$

See [8], Section 5.5 and p. 190. From [8], (8.22.8) on p. 200 we have

$$
h_{n}(t)=\frac{2^{1 / 2}}{\left(\pi\left(n+\frac{1}{2}\right)\right)^{1 / 4}} \cos \left[2\left(\pi\left(n+\frac{1}{2}\right)\right)^{1 / 2} t-\frac{1}{2} \pi n\right]+O\left(\left(n+\frac{1}{2}\right)^{-3 / 4}\right),
$$

where the $O$ holds uniformly locally in $t \in \mathbb{R}$. Also, from the table on p. 700 in [9] (in which we have $\left.h_{n}(t)=(2 \pi)^{1 / 4} \mathcal{H}_{n}(t \sqrt{2 \pi})\right)$, we get

$$
\begin{aligned}
& h_{n}(t)=O\left((n+1)^{-1 / 4}\right), \quad-\frac{n}{\sqrt{2 \pi}} \leq t \leq \frac{n}{\sqrt{2 \pi}}, \\
& h_{n}(t)=O\left((n+1)^{-1 / 12}\right), \quad t \in \mathbb{R} .
\end{aligned}
$$

The Gaussian window STFT of the Hermite functions can be computed by using properties of the Bargmann transform, see [2], p. 40 and [3], Section 3.4. With $f \in L^{2}(\mathbb{R})$, the Bargmann transform $B f$ of $f$ is defined as

$$
(B f)(z)=e^{-\frac{1}{2} \pi z^{2}} \int_{-\infty}^{\infty} f(t) 2^{1 / 4} e^{-\pi(t-z)^{2}} d t, \quad z . \in \mathbb{C} .
$$

There holds for $f \in L^{2}(\mathbb{R})$

$$
\left(f, g_{x, y}\right)=e^{-\frac{1}{2} \pi\left(x^{2}+y^{2}\right)-\pi i x y}(B f)(x-i y), \quad x, y \in \mathbb{R} .
$$

From the generating formula, [2], (1.86) on p. 54, for the Hermite functions and orthonormality it follows that

$$
\left(B h_{n}\right)(z)=\frac{\left(\pi^{1 / 2} z\right)^{n}}{(n !)^{1 / 2}}, \quad n=0,1, \ldots, \quad z \in \mathbb{C} .
$$

Consequently, one has

$$
\left(h_{n}, g_{x, y}\right)=e^{-\frac{1}{2} \pi\left(x^{2}+y^{2}\right)-\pi i x y} \frac{\left(\pi^{1 / 2}(x-i y)\right)^{n}}{(n !)^{1 / 2}}, \quad n=0,1, \ldots, \quad x, y \in \mathbb{R} .
$$

We then compute, for instance,

$$
\begin{aligned}
\left\|h_{n}\right\|_{S_{0}} & =\int_{-\infty}^{\infty} \int_{-\infty}^{\infty} e^{-\frac{1}{2} \pi\left(x^{2}+y^{2}\right)} \frac{\left(\pi^{1 / 2}\left(x^{2}+y^{2}\right)^{1 / 2}\right)^{n}}{(n !)^{1 / 2}} d x d y \\
& =2^{\frac{1}{2} n+1} \frac{\Gamma\left(\frac{1}{2} n+1\right)}{(n !)^{1 / 2}}, \quad n=0,1, \ldots
\end{aligned}
$$


in which we have used polar coordinates $x+i y=r e^{i \varphi}$ to evaluate the double integral.

Mehler's formula can be found in [8], Problem 23 on p. 380. In terms of the Hermite functions $h_{n}$ it takes the form as can be found in [2], (1.87) on p. 55: for $t, s \in \mathbb{C}, w \in \mathbb{C}$, $|w|<1$ there holds

$$
\sum_{n=0}^{\infty} w^{n} h_{n}(t) h_{n}(s)=\left(\frac{2}{1-w^{2}}\right)^{1 / 2} \exp \left(\frac{4 \pi t s w-\pi\left(t^{2}+s^{2}\right)\left(1+w^{2}\right)}{1-w^{2}}\right) .
$$

\section{Proof that $C\left(\frac{1}{4}\right) \subset S_{0} \subset C\left(-\frac{1}{4}\right)$}

We shall first show that $C\left(\frac{1}{4}\right) \subset S_{0}$. It follows from Stirling's formula $\Gamma(x+1)=$ $x^{x+1 / 2} e^{-x} \sqrt{2 \pi}\left(1+O\left(\frac{1}{x}\right)\right)$, see [10], 6.1.37-38 on p. 257, and (2.10) that

$$
\left\|h_{n}\right\|_{S_{0}}=2\left(\frac{1}{2} \pi n\right)^{1 / 4}\left(1+O\left(n^{-1}\right)\right), \quad n=0,1, \ldots .
$$

Since $S_{0}$ is a Banach space, we have for $f \in \mathcal{S}^{\prime}$ that

$$
\sum_{n=0}^{\infty}\left|\left(f, h_{n}\right)\right|(n+1)^{1 / 4}<\infty \Rightarrow f=\sum_{n=0}^{\infty}\left(f, h_{n}\right) h_{n} \in S_{0} .
$$

Hence, when $f \in C\left(\frac{1}{4}\right)$ we have that $f \in S_{0}$, and the Hermite series of $f$ converges in the sense of $S_{0}$ to $f$.

We next show that $S_{0} \subset C_{0}\left(-\frac{1}{4}\right)$. We let $f \in L^{2}(\mathbb{R})$, and we apply the inversion formula for the STFT, see [3], Corollary 3.2.3 on p. 44, to obtain

$$
\begin{aligned}
\left|\left(f, h_{n}\right)\right| & =\left|\int_{-\infty}^{\infty} \int_{-\infty}^{\infty}\left(f, g_{x, y}\right)\left(g_{x, y}, h_{n}\right) d x d y\right| \\
& \leq \int_{-\infty}^{\infty} \int_{-\infty}^{\infty}\left|\left(f, g_{x, y}\right)\right| e^{-\frac{1}{2} \pi\left(x^{2}+y^{2}\right)} \frac{\left(\pi^{1 / 2}\left(x^{2}+y^{2}\right)^{1 / 2}\right)^{n}}{(n !)^{1 / 2}} d x d y,
\end{aligned}
$$

where we have used (2.9). Assume that $f \in S_{0}$. We must show that $\sum_{n}\left|\left(f, h_{n}\right)\right|(n+$ $1)^{-1 / 4}<\infty$. To that end we shall prove below that there is a $C>0$ such that

$$
F(v):=\sum_{n=0}^{\infty} \frac{v^{n / 2}}{(n !)^{1 / 2}(n+1)^{1 / 4}} \leq C e^{\frac{1}{2} v}, \quad v \geq 0 .
$$

It follows from (3.4) that

$$
\sum_{n=0}^{\infty}\left|\left(f, h_{n}\right)\right|(n+1)^{-1 / 4} \leq C \int_{-\infty}^{\infty} \int_{-\infty}^{\infty}\left|\left(f, g_{x, y}\right)\right| d x d y=C\|f\|_{S_{0}},
$$

which implies that $f \in C\left(-\frac{1}{4}\right)$. 
We shall now show that (3.4) holds for some $C>0$. To that end we prove that there are $c_{1}>0, c_{2}>0$ such that

$$
\frac{v^{x} e^{-v}}{\Gamma(x+1)} \leq \frac{1}{\sqrt{2 \pi x}} \cdot \begin{cases}\exp \left(-\frac{c_{1}}{x}(v-x)^{2}\right), & \frac{1}{2} v \leq x \leq 2 v \\ \exp \left(-c_{2}|v-x|\right) & 0 \leq x \leq \frac{1}{2} v \text { or } x \geq 2 v\end{cases}
$$

To show (3.6), we first note that from the inequality $\Gamma(x+1) \geq x^{x} e^{-x} \sqrt{2 \pi x}, x \geq 0$, see [11], bottom half of p. 251, we have that

$$
\frac{v^{x} e^{-v}}{\Gamma(x+1)} \leq \frac{1}{\sqrt{2 \pi x}} \exp \left[x\left(\ln \frac{v}{x}+1-\frac{v}{x}\right)\right], \quad x, v \geq 0
$$

The function $w>0 \rightarrow r(w):=\ln w+1-w$ is smooth and concave, and has its maximum 0 at $w=1$. Consequently, there are $c_{1}>0, c_{2}>0$ such that

$$
\begin{array}{ll}
r(w) \leq-c_{1}(w-1)^{2}, & \frac{1}{2} \leq w \leq 2 \\
r(w) \leq-c_{2}|w-1|, & 0<w \leq \frac{1}{2} \text { or } w \geq 2
\end{array}
$$

From this (3.6) follows. Therefore, when $v \geq 1$, we have

$$
\begin{aligned}
e^{-\frac{1}{2} v} F(v)= & \sum_{n=0}^{\infty}\left(\frac{v^{n} e^{-v}}{n !}\right)^{1 / 2} \frac{1}{(n+1)^{1 / 4}} \\
\leq & e^{-\frac{1}{2} v}+\left(\sum_{1 \leq n \leq \frac{1}{2} v}+\sum_{n \geq 2 v}\right)\left(\frac{1}{2 \pi n(n+1)}\right)^{1 / 4} \exp \left(-\frac{1}{2} c_{2}|v-n|\right) \\
& +\sum_{\frac{1}{2} v \leq n \leq 2 v}\left(\frac{1}{2 \pi n(n+1)}\right)^{1 / 4} \exp \left(-\frac{c_{1}}{2 n}(v-n)^{2}\right)
\end{aligned}
$$

The first term on the second line of (3.10), $\exp \left(-\frac{1}{2} v\right)$, is bounded in $v \geq 0$. The second term is bounded by $(4 / \pi)^{1 / 4}\left(1-\exp \left(-\frac{1}{2} c_{2}\right)\right)^{-1} \exp \left(-\frac{1}{4} c_{2} v\right)$, and the term on the last line is bounded by

$$
\begin{aligned}
& \left(\frac{2}{\pi v(v+2)}\right)^{1 / 4} \sum_{n=-\infty}^{\infty} \exp \left(-\frac{1}{4} c_{1} v^{-1}(v-n)^{2}\right) \\
& \quad=\left(\frac{2}{\pi v(v+2)}\right)^{1 / 4}\left(\frac{4 \pi v}{c_{1}}\right)^{1 / 2} \sum_{m=-\infty}^{\infty} \exp \left[-4 \pi^{2} m^{2} v / c_{1}+2 \pi i m v\right] \\
& \quad \leq\left(\frac{32 \pi}{c_{1}^{2}} \frac{v}{v+2}\right)^{1 / 4} \sum_{m=-\infty}^{\infty} \exp \left(-4 \pi^{2} m^{2} v / c_{1}\right) \\
& \quad \leq\left(\frac{32 \pi}{c_{1}^{2}}\right)^{1 / 4} \sum_{m=-\infty}^{\infty} \exp \left(-4 \pi^{2} m^{2} / c_{1}\right)
\end{aligned}
$$

when $v \geq 1$. Here we have used the Poisson summation formula for the first identity in (3.11). This shows (3.4). 


\section{Notes.}

1. In a similar fashion one can prove the existence of a $D>0$ such that

$$
\sum_{n=0}^{\infty}\left|\left(f, h_{n}\right)\right| \leq D \int_{-\infty}^{\infty} \int_{-\infty}^{\infty}\left|\left(f, g_{x, y}\right)\right|\left(1+x^{2}+y^{2}\right)^{1 / 4} d x d y
$$

holds for any $f \in L^{2}(\mathbb{R})$. Furthermore, many variations on this theme are possible.

2. From what we show in Section 5 it follows that $C\left(-\frac{1}{4}\right) \not \subset S_{0}$. Nevertheless, the members of $C\left(-\frac{1}{4}\right)$ are reasonably well-behaved functions. Indeed, it follows from (2.4) and/or (2.5) that the function $f$ and its Fourier transform $\mathcal{F} f$,

$$
f=\sum_{n=0}^{\infty}\left(f, h_{n}\right) h_{n}, \quad \mathcal{F} f=\sum_{n=0}^{\infty} i^{n}\left(f, h_{n}\right) h_{n}
$$

are continuous, the series being locally uniformly convergent when $f \in C\left(-\frac{1}{4}\right)$.

\section{Proof that $S_{0} \not \subset C(\gamma), \gamma>-\frac{1}{4}$}

We shall now show that $S_{0} \not \subset C(\gamma)$ when $-\frac{1}{4}<\gamma<\frac{1}{4}$. For this we use Mehler's formula (2.11) with $s=0$ and $w=i v, 0 \leq v<1$, so that for $0 \leq v<1, t \in \mathbb{R}$

$$
\sum_{n=0}^{\infty} i^{n} v^{n} h_{n}(0) h_{n}(t)=\left(\frac{2}{1+v^{2}}\right)^{1 / 2} \exp \left(-\pi \frac{1-v^{2}}{1+v^{2}} t^{2}\right) .
$$

We observe from [8], 5.5.4-5 on p. 106 that $h_{2 m+1}(0)=0, m=0,1, \ldots$, while

$$
(-1)^{m} h_{2 m}(0)=\left(2^{-2 m+1 / 2} \frac{(2 m) !}{m ! m !}\right)^{1 / 2}, \quad m=0,1, \ldots
$$

From Appendix A it follows that

$$
\left(\frac{2}{\pi\left(m+\frac{1}{2}\right)}\right)^{1 / 4} \leq(-1)^{m} h_{2 m}(0) \leq\left(\frac{2}{\pi\left(m+\frac{1}{4}\right)}\right)^{1 / 4}, \quad m=0,1, \ldots .
$$
results

Now let $\alpha \in\left(\frac{1}{2}, 1\right]$, multiply (4.1) by $(1-v)^{\alpha-1}$, and integrate over $v \in[0,1)$. There

$$
\begin{aligned}
& \sum_{m=0}^{\infty}(-1)^{m} h_{2 m}(0) B(\alpha, 2 m+1) h_{2 m}(t) \\
& \quad=\int_{0}^{1}\left(\frac{2}{1+v^{2}}\right)^{1 / 2} \exp \left(-\pi \frac{1-v^{2}}{1+v^{2}} t^{2}\right)(1-v)^{\alpha-1} d v=: f_{\alpha}(t), \quad t \in \mathbb{R}
\end{aligned}
$$

where for $m=0,1, \ldots$

$$
B(\alpha, 2 m+1)=\int_{0}^{1}(1-v)^{\alpha-1} v^{2 m} d v=\frac{\Gamma(\alpha) \Gamma(2 m+1)}{\Gamma(2 m+1+\alpha)} .
$$


In (4.4) we have interchanged the summation over $m=0,1, \ldots$ and the integration over $v \in[0,1)$. This is justifiable by (2.4) and/or (2.5), (4.3), and the fact that the integrand in (4.5) is non-negative while by Appendix A

$$
\Gamma(\alpha)(2 m+1)^{-\alpha} \leq B(\alpha, 2 m+1) \leq \Gamma(\alpha)\left(2 m+\frac{1}{2} \alpha+\frac{1}{2}\right)^{-\alpha} .
$$

We also see from (4.3) and (4.6) that $f_{\alpha} \in C(\gamma)$ if and only if $\alpha>\frac{3}{4}+\gamma$.

We shall now show that $f_{\alpha}$ in (4.4), with $\alpha>\frac{1}{2}$, is in $S_{0}$. To that end we compute for $\beta>0$ the Gaussian window STFT of $\exp \left(-\pi \beta t^{2}\right)$ as

$$
\begin{aligned}
& \int_{-\infty}^{\infty} e^{-\pi \beta t^{2}} 2^{1 / 4} e^{-\pi(t-x)^{2}-2 \pi i y t} d t \\
& =2^{1 / 4} \frac{1}{\sqrt{1+\beta}} \exp \left[\frac{-\pi \beta}{1+\beta} x^{2}-\frac{\pi}{1+\beta} y^{2}-\frac{2 \pi i}{1+\beta} x y\right], \quad x, y \in \mathbb{R},
\end{aligned}
$$

and the $S_{0}$-norm of $\exp \left(-\pi \beta t^{2}\right)$ as

$$
\int_{-\infty}^{\infty} \int_{-\infty}^{\infty} \frac{2^{1 / 4}}{\sqrt{1+\beta}} \exp \left[\frac{-\pi \beta}{1+\beta} x^{2}-\frac{\pi}{1+\beta} y^{2}\right] d x d y=2^{1 / 4}\left(\frac{1+\beta}{\beta}\right)^{1 / 2}
$$

It follows that

$$
\left\|f_{\alpha}\right\|_{S_{0}} \leq \int_{0}^{1}\left(\frac{2}{1+v^{2}}\right)^{1 / 2}(1-v)^{\alpha-1} 2^{1 / 4}\left(\frac{2}{1-v^{2}}\right)^{1 / 2} d v<\infty
$$

since $\alpha>\frac{1}{2}$. Conversely, when we consider $f_{\alpha}$ in (4.4) with $\alpha>0$, we have that

$$
\int_{-\infty}^{\infty} f_{\alpha}(t) d t=\int_{0}^{1}\left(\frac{2}{1+v}\right)^{1 / 2}(1-v)^{\alpha-3 / 2} d v<\infty
$$

if and only if $\alpha>\frac{1}{2}$. Thus, $f_{\alpha} \in S_{0}$ if and only if $\alpha>\frac{1}{2}$.

We conclude that for $\gamma>-\frac{1}{4}$ we have $f_{\alpha} \in S_{0} \backslash C(\gamma)$ if and only if $\frac{1}{2}<\alpha \leq \frac{3}{4}+\gamma$. In particular, $S_{0} \backslash C(\gamma)$ is non-empty when $\gamma>-\frac{1}{4}$. At this point it is interesting to ask whether perhaps the Hermite series for $f_{\alpha}$, see (4.4), converges in $S_{0}$-sense when $\frac{1}{2}<\alpha \leq$ 1. This is indeed so, but the proof is quite technical and so we omit it.

\section{Proof that $C(\gamma) \not \subset S_{0}, \gamma<\frac{1}{4}$}

We start by noting that $C\left(\gamma_{1}\right) \subset C\left(\gamma_{2}\right)$ when $\gamma_{1}>\gamma_{2}$, whence it is sufficient to consider $0<\gamma<\frac{1}{4}$. We shall consider $f \in L^{2}(\mathbb{R})$ of the form

$$
f=\sum_{m=0}^{\infty} c_{m} h_{K_{m}}
$$


where we take $K_{m}=A^{m}$ with integer $A \geq 4$ and $c_{m}=\left(K_{m}+1\right)^{-1 / 4}$. This $f$ satisfies

$$
\sum_{n=0}^{\infty}\left|\left(f, h_{n}\right)\right|(n+1)^{\gamma}=\sum_{m=0}^{\infty}\left(A^{m}+1\right)^{\gamma-1 / 4}<\infty .
$$

Hence, $f \in C(\gamma)$ for $\gamma<\frac{1}{4}$, and certainly $f \in L^{2}(\mathbb{R})$.

We compute, using (2.9), for this $f$

$$
\begin{aligned}
\|f\|_{S_{0}} & =\int_{-\infty}^{\infty} \int_{-\infty}^{\infty} e^{-\frac{1}{2} \pi\left(x^{2}+y^{2}\right)}\left|\sum_{m=0}^{\infty} c_{m} \frac{\left(\pi^{1 / 2}(x-i y)\right)^{K_{m}}}{\left(K_{m} !\right)^{1 / 2}}\right| d x d y \\
& =\frac{1}{2 \pi} \int_{0}^{\infty} \int_{0}^{2 \pi}\left|\sum_{m=0}^{\infty} c_{m} f_{m}(v) e^{-i K_{m} \varphi}\right| d v d \varphi
\end{aligned}
$$

where we have used polar coordinates $x+i y=r e^{i \varphi}$ and subsequently substituted $v=\pi r^{2}$, and where $f_{m}$ is defined by

$$
f_{m}(v)=\left(\frac{v^{K_{m}} e^{-v}}{K_{m} !}\right)^{1 / 2}, \quad m=0,1, \ldots, v \geq 0 .
$$

Since $A \geq 4, K_{m}=A^{m}$, we have that the intervals $\left(\frac{1}{2} K_{m}, 2 K_{m}\right)$ are non-overlapping. Consequently,

$$
\begin{aligned}
\|f\|_{S_{0}} & \geq \frac{1}{2 \pi} \sum_{m=0}^{\infty} \int_{\frac{1}{2} K_{m}}^{2 K_{m}} \int_{0}^{2 \pi}\left(c_{m} f_{m}(v)-\sum_{n \neq m} c_{n} f_{n}(v)\right) d v d \varphi \\
& =\sum_{m=0}^{\infty} I_{m m}-\sum_{m=0}^{\infty} \sum_{n=0, n \neq m}^{\infty} I_{m n} \\
& =\sum_{m=0}^{\infty} I_{m m}-\sum_{n=0}^{\infty} \sum_{m=0, m \neq n}^{\infty} I_{m n}
\end{aligned}
$$

where

$$
I_{m n}=\int_{\frac{1}{2} K_{m}}^{2 K_{m}} c_{n} f_{n}(v) d v, \quad m, n=0,1, \ldots
$$

For $m=0,1, \ldots$, there holds

$$
I_{m m}=\int_{0}^{\infty} c_{m} f_{m}(v) d v-\int_{v \notin\left(\frac{1}{2} K_{m}, 2 K_{m}\right)} c_{m} f_{m}(v) d v,
$$

while for $n=0,1, \ldots$, there holds

$$
\sum_{m=0, m \neq n}^{\infty} I_{m n}=\sum_{m=0, m \neq n}^{\infty} \int_{\frac{1}{2} K_{m}}^{2 K_{m}} c_{n} f_{n}(v) d v \leq \int_{v \notin\left(\frac{1}{2} K_{n}, 2 K_{n}\right)} c_{n} f_{n}(v) d v
$$


Hence,

$$
\|f\|_{S_{0}} \geq \sum_{m=0}^{\infty} \int_{0}^{\infty} c_{m} f_{m}(v) d v-2 \sum_{m=0}^{\infty} \int_{v \notin\left(\frac{1}{2} K_{m}, 2 K_{m}\right)} c_{m} f_{m}(v) d v .
$$

Now from (5.4) and (3.6) we see that

$$
\int_{v \notin\left(\frac{1}{2} K_{m}, 2 K_{m}\right)} f_{m}(v) d v \leq \frac{1}{\sqrt{2 \pi K_{m}}} \int_{-\infty}^{\infty} \exp \left(-\frac{1}{2} c_{2}\left|v-K_{m}\right|\right) d v=\frac{4}{c_{2} \sqrt{2 \pi K_{m}}}
$$

With $c_{m}=\left(K_{m}+1\right)^{-1 / 4}, K_{m}=A^{m}, A \geq 4$, we then see that the second series at the right-hand side of (5.9) is finite. On the other hand, we have from (5.4)

$$
\int_{0}^{\infty} f_{m}(v) d v=\left(\frac{1}{K_{m} !}\right)^{1 / 2} \int_{0}^{\infty} v^{\frac{1}{2} K_{m}} e^{-\frac{1}{2} v} d v=2\left(\frac{2^{K_{m}} \Gamma^{2}\left(\frac{1}{2} K_{m}+1\right)}{\Gamma\left(K_{m}+1\right)}\right)^{1 / 2} .
$$

By Appendix A there holds for $y>0$

$$
\frac{2^{y} \Gamma^{2}\left(\frac{1}{2} y+1\right)}{\Gamma(y+1)} \geq \sqrt{\frac{1}{2} \pi y} .
$$

Therefore

$$
\int_{0}^{\infty} f_{m}(v) d v \geq 2^{3 / 4} \pi^{1 / 4} K_{m}^{1 / 4}
$$

whence the first series at the right-hand side of (5.9) equals $\infty$ since $c_{m}=\left(K_{m}+1\right)^{-1 / 4}$. Therefore, $\|f\|_{S_{0}}=\infty$ and thus $f \notin S_{0}$. Since $f \in C(\gamma)$ when $\gamma<\frac{1}{4}$, we see that $C(\gamma) \not \subset S_{0}$, and the proof is complete.

\section{Appendix}

\section{A. Appendix. Some Lemmas about $B$-Functions}

In the main text we needed for $m=0,1, \ldots, \alpha \in(0,1), y>0$

$$
\begin{gathered}
\frac{1}{\sqrt{\pi\left(m+\frac{1}{2}\right)}} \leq 2^{-2 m} \frac{(2 m) !}{m ! m !} \leq \frac{1}{\sqrt{\pi\left(m+\frac{1}{4}\right)}}, \\
\Gamma(\alpha)(2 m+1)^{-\alpha} \leq B(\alpha, 2 m+1) \leq \Gamma(\alpha)\left(2 m+\frac{1}{2} \alpha+\frac{1}{2}\right)^{-\alpha}, \\
\frac{2^{y} \Gamma^{2}\left(\frac{1}{2} y+1\right)}{\Gamma(y+1)}=\frac{1}{2}(y+1) B\left(\frac{1}{2}, \frac{1}{2} y+1\right) \geq \sqrt{\frac{1}{2} \pi y},
\end{gathered}
$$


where the $B$-function is given as

$$
B(\alpha, x+1)=\int_{0}^{1}(1-v)^{\alpha-1} v^{x} d v=\frac{\Gamma(\alpha) \Gamma(x+1)}{\Gamma(x+1+\alpha)} .
$$

We first observe that

$$
2^{-2 m} \frac{(2 m) !}{m ! m !}=\frac{\Gamma\left(m+\frac{1}{2}\right)}{\Gamma(m+1) \Gamma\left(\frac{1}{2}\right)}=\frac{1}{\pi} B\left(\frac{1}{2}, m+\frac{1}{2}\right),
$$

and that for $x>-1$

$$
\frac{2^{2 x} \Gamma^{2}(x+1)}{\Gamma(2 x+1)}=2^{2 x}(2 x+1) B(x+1, x+1),
$$

while

$$
\begin{aligned}
2^{2 x} B(x+1, x+1) & =2^{2 x} \int_{0}^{1}(t(1-t))^{x} d t=\int_{0}^{1}\left(1-(1-2 t)^{2}\right)^{x} d t \\
& =\frac{1}{2} \int_{0}^{1}(1-s)^{x} s^{-1 / 2} d s=\frac{1}{2} B\left(\frac{1}{2}, x+1\right) .
\end{aligned}
$$

It thus suffices to show that for $\alpha \in(0,1], x>-1$

$$
\Gamma(\alpha)(x+1)^{-\alpha} \leq B(\alpha, x+1) \leq \Gamma(\alpha)\left(x+\frac{1}{2} \alpha+\frac{1}{2}\right)^{-\alpha} .
$$

We have by the substitution $v=e^{-t}, t \in[0, \infty)$

$$
\begin{aligned}
B(\alpha, x+1) & =\int_{0}^{1}(1-v)^{\alpha-1} v^{x} d v=\int_{0}^{\infty}\left(1-e^{-t}\right)^{\alpha-1} e^{-(x+1) t} d t \\
& =\int_{0}^{\infty} t^{\alpha-1}\left(\frac{\sinh (t / 2)}{t / 2}\right)^{\alpha-1} e^{-\left(x+\frac{1}{2} \alpha+\frac{1}{2}\right) t} d t .
\end{aligned}
$$

Since for $t \geq 0, b>0$

$$
1-e^{-t} \leq t, \quad \frac{\sinh (t / 2)}{t / 2} \geq 1, \quad \int_{0}^{\infty} t^{\alpha-1} e^{-b t} d t=b^{-\alpha} \Gamma(\alpha),
$$

we get (A.8).

\section{Acknowledgments}

It is a pleasure to thank Hans Feichtinger and Franz Luef for their comments on earlier versions of this article. 


\section{References}

[1] Feichtinger, H. G. and Strohmer, T., Eds. (1998). Gabor Analysis and Algorithms - Theory and Applications, Boston, Birkhäuser.

[2] Folland, G. B. (1989). Harmonic Analysis in Phase Space, Princeton University Press, Princeton

[3] Gröchenig, K. (2000). Foundations of Time-Frequency Analysis, Boston, Birkhäuser.

[4] Simon, B. (1971). Distributions and their Hermite expansions, J. Math. Phys. 12(1), 140-148.

[5] Hogan, J. H. and Lakey, J. D. (2005). Time-Frequency and Time-Scale Methods, Boston, Birkhäuser.

[6] Berger, C. A. and Coburn, L. A. (1986). Toeplitz operators and quantum mechanics, J. Funct. Anal. 68, 273-299.

[7] Janssen, A. J. E. M. (1982). Bargmann transform, Zak transform, and coherent states, J. Math. Phys. 23(5), $720-731$.

[8] Szegö, G. (1975). Orthogonal polynomials, Amer. Math. Soc. 4th ed., Providence.

[9] Askey, R. and Wainger, S. (1965). Mean convergence of expansions in Laguerre and Hermite series, Amer. J. Math. 87, 695-708.

[10] Abramowitz, M. and Stegun, I. A. (1970). Handbook of Mathematical Functions, New York, Dover, 9th printing.

[11] Whittaker, E. T. and Watson, G. N. (1962). A Course of Modern Analysis, Cambridge University Press, Cambridge, 4th ed.

Received December 22, 2004

Revision received July 01, 2005

Philips Research Laboratories, Eindhoven, 5656 AA Eindhoven, The Netherlands 